\title{
¿Existen problemas estructurales irresolubles? Una cuestión abierta
}

\author{
Are there unsolvable structural problems? \\ An open question
}

J. Antuña ${ }^{(*)}, \underline{M}$. Vázquez ${ }^{(*)}$

\section{RESUMEN}

Galileo es el primer autor occidental que señaló la imposibilidad de que una figura creciera indefinidamente por semejanza, definiendo el concepto de un tamaño insuperable para cualquier estructura mecánica sujeta a la acción de su propio peso. Si tal tamaño insuperable existe para un problema estructural, tal problema sería irresoluble para tamaños mayores. La cuestión no ha sido contestada hasta la fecha, a pesar de múltiples investigaciones según dos líneas contrapuestas: por un lado, la determinación de formas de tensión constante no limitadas a un tamaño concreto; por otro, la determinación de tamaños insuperables para problemas estructurales bien definidos. Aquí se presentan dos hipótesis relacionadas que podrían encauzar la búsqueda de una respuesta definitiva.

$400-42$

Palabras clave: diseño de estructuras, tamaño insuperable, coste estructural, alcance estructural, formas óptimas.

\section{SUMMARY}

Galileo is the first Western author who pointed out the impossibility of that a similar figure grows indefinitely, by defining the concept of an insuperable size for any structure subject to the mechanical action of self-weight. If such insuperable size exists for a structural problem, such a problem would be unsolvable for greater sizes. The matter has not been answered until date, despite multiple researches along two conflicting lines: firstly, the determination of forms of constant stress whit no limit in size, and secondly, the determination of insuperable sizes for well defined structural problems. Here two related hypotheses are presented which could guide the search for a definitive answer.

Keywords: structural design, insuperable size, structural cost, structural scope, optimal shapes. 


\section{PROBLEMAS ESTRUCTURALES}

Un problema estructural puede definirse de forma abstracta describiendo la geometría de una carga útil que debe ser soportada respecto de una sustentación, con geometría igualmente definida. Resolverlo consiste en encontrar para un material dado de antemano la geometría de una figura que de lugar a una estructura que sea resistente, rígida y estable para el problema. Generalmente existen varias soluciones pero, desde siempre, hay un "deseo" implícito de determinar aquella solución que sea óptima, es decir, cuyo coste físico sea el menor entre el conjunto de soluciones aceptables.

En primera aproximación, el coste físico puede representarse por el peso propio de la estructura ya que casi cualquier coste físico imaginable tiene una relación monótona -no necesariamente proporcional-con el peso propio. De este modo, la búsqueda de las estructuras de mínimo peso representa el primer y principal paso en cualquier teoría de diseño. Aquí, por razones de brevedad nos contentaremos con un manejo informal de la noción de problema estructural, a sabiendas de que es posible y deseable su formalización. Detalles adicionales pueden consultarse en Cervera $(1,2)$.

\section{LOS TAMAÑOS INSUPERABLES DE GALILEO}

\section{PROPOSICIÓN VII}

Entre los prismas o cilindros pesados [gravi] y semejantes, hay uno y solo uno que llega a encontrarse (a consecuencia de su propio peso) en un estado límite entre romperse y mantenerse todavía entero, de modo que todo aquel que sea más grande, incapaz de sostener su propio peso, se romperá, mientras que todo el que sea más pequeño opondrá alguna resistencia a la fuerza que se haga para romperlo. [...]

De lo que se ha demostrado hasta el momento, como podéis ver, se infiere la imposibilidad de poder, no solo en el arte sino en la misma naturaleza, aumentar los mecanismos hasta dimensiones inmensas, de modo que sería imposible fabricar naves, palacios o templos enormes, de tal forma que sus remos, patios, vigas, cerrojos $y$, en suma, todas sus partes constituyentes, pudiesen sostenerse. Así, tampoco podría la naturaleza hacer árboles de un tamaño desmesurado, ya que sus ramas acabarían por venirse abajo bajo su propio peso. Sería imposible, igualmente, construir estructuras óseas de hombres, caballos u otros animales, que pudiesen mantenerse y realizar sus propios menesteres, a no ser que se utilizara un material más duro y re- sistente que el normal, en caso de que no se les agrandara tales huesos de modo tan desproporcionado que la figura y aspecto del animal en cuestión llegase a ser algo monstruosamente grande; lo cual, tal vez, intuyó nuestro sagaz Poeta cuando, describiendo un grandísimo gigante, decía:

Imposible reconocer su altura,

tan desmesuradamente grande es su grosor

Galileo, Discorsi

La que ha venido en Ilamarse ley de los Cubos y los cuadrados, formulada por Galileo por vez primera en sus Discorsi, es una proposición muy general sobre la imposibilidad del crecimiento indefinido por semejanza de una figura sujeta a condiciones que atañen simultáneamente a su superficie y a su volumen.

El caso mecánico es particularmente simple de explicar: la resistencia de estructuras semejantes, proporcional al área, crece con el cuadrado de su tamaño, mientras que su propio peso lo hace con el cubo. Si una estructura, que es segura para un tamaño y carga útil dados, crece por semejanza, verá aumentar su peso propio más deprisa que su resistencia, alcanzando para algún tamaño determinado una situación insuperable, en la que la estructura colapsa por su propio peso sin necesidad de carga útil adicional.

La resistencia, medida en términos de tensión (una fuerza por unidad de superficie), depende de un flujo. Por su parte, el peso es un stock, medido por unidad de volumen. Las condiciones de equilibrio mecánico pueden describirse como un compromiso entre flujos y stocks; esta idea puede generalizarse a cualquier sistema físico cuya estabilidad resulta de un compromiso entre ambos: así su utilidad ha sido explorada en campos tan dispares como la conducción de calor, la cosmología cuántica o el transporte en sistemas urbanos.

Debe notarse que la imposibilidad mencionada solo se refiere a figuras semejantes de distinto tamaño. El propio Galileo, a renglón seguido de su proposición VII, comenzó a explorar en que condiciones podrían superarse tales tamaños insuperables. Su respuesta ofrece dos tipos de soluciones en el caso mecánico: por una lado, utilizar materiales más resistentes e igual o menos densos; por otro, renunciar a la semejanza formal y variar la forma de la figura. La primera solución es una mera maniobra de distracción: un material más resistente tan solo aumenta el tamaño insuperable, sin acabar con su existencia. La segunda solución es mucho más prometedora: permite abrigar la esperanza de que exista, para 
cada tamaño, una forma óptima capaz no solo de soportarse a sí misma, también de soportar carga adicional; en último extremo, permite especular con la posibilidad de que cualquier problema estructural sea resoluble para cualquier tamaño, sin más que encontrar la figura apropiada.

Podemos dar cuerpo a esa especulación en la forma de una Hipótesis Optimista:

Entre todas las figuras estructurales no semejantes pero de igual tamaño, y que resuelven un mismo problema estructural, existe al menos una que es capaz de soportar su propio peso y alguna carga útil.

En la siguiente discusión cobrará importancia el concepto de coste: pues desde un punto de vista práctico tanto da disponer de una solución de coste impagable que no tenerla. La definición estándar de la termodinámica para rendimiento es [1]:

$$
\text { Rendimiento }=\frac{\text { Cantidad útil }}{\text { Cantidad total }}
$$

La Segunda Ley es siempre de aplicación en cualquier sistema ergódico (3), de manera que en un sistema aislado el rendimiento es siempre menor que la unidad. El coste es, simplemente, el inverso del rendimiento [2]:

\section{[2] Coste $=\frac{1}{\text { Rendimiento }}=\frac{\text { Cantidad total }}{\text { Cantidad útil }}$}

y para sistemas aislados, el coste es siempre mayor que la unidad.

La Segunda Ley admite una reinterpretación mecánica, y la Mecánica una reinterpretación termodinámica, digna de mención: una estructura se diseña para soportar carga útil. La estructura ideal es la que no pesa, dedicando toda su resistencia a soportar carga útil. En tal condición ideal (análoga a la de reversibilidad), el rendimiento y el coste son la unidad. Pero una estructura real pesa inevitablemente así que parte de la resistencia debe emplearse en sostener su propio peso, y el rendimiento resulta menor que la unidad (y el coste mayor que ella). En los casos más frecuentes el rendimiento estructural disminuye monótonamente con el tamaño. $Y$ puesto que las estructuras construidas con los nuevos materiales de la Revolución Industrial son casi siempre estructuralmente pequeñas, los rendimientos pueden acercarse a la unidad'. En términos prácticos: el peso propio de tales estructuras es despreciable, y despreciarlo es la práctica habitual en su cálculo y diseño iniciales. Sin embargo, cualitativamente, el rendimiento estructural es siempre menor que la unidad, y será tanto menor cuanto más grande sea la estructura. La proposición VII, traducida a nuestro ac- tual vocabulario, dice que para estructuras semejantes, existe un tamaño finito para el que el rendimiento es nulo (y el coste infinito). A partir de ahí podemos formular una Hipótesis Pesimista general:

Para cualquier problema estructural existe un tamaño insuperable finito para el que cualquier forma estructural colapsa bajo su propio peso.

La Hipótesis Pesimista (HP) es razonablemente refutable: basta especificar un problema y una figura en el que el tamaño insuperable sea infinito, es decir, que para cualquier tamaño finito el rendimiento de la figura en cuestión, aunque pequeño, no sea nulo. La Hipótesis Optimista (HO), sin embargo, no es razonablemente refutable, pues obligaría a mostrar para al menos un problema concreto que todas las figuras posibles o bien no resuelven el problema o bien, si lo hacen, tienen rendimiento nulo para un tamaño finito.

Pasemos ahora a examinar el relativo mérito de los casos que apoyan cada una de ambas hipótesis.

\section{TAMAÑOS INSUPERABLES}

\subsection{El peso suspendido de un cable}

Si la sección del cable es constante su resistencia es el producto de su máxima tensión, $\boldsymbol{f}$, por el área de su sección, $\boldsymbol{A}$. Para una longitud $L$, su peso $P$ es proporcional a su volumen, $\rho L \boldsymbol{A}$, siendo $\rho$ su peso específico. Con un peso $Q$ tenemos [3]:

$\mathbf{f A}=\rho L \boldsymbol{A}+Q=P+Q$

Existe un tamaño límite más allá del cual, aun sin peso $(Q=0)$, el cable resulta inseguro [4]:

$L \leq \mathscr{L}=\frac{\boldsymbol{f}}{\rho}$

El tamaño límite del cable no depende de su sección y como se ve es una propiedad del material: ese cociente ha recibido el nombre de alcance del material, $\propto$. El alcance del esquema "cable vertical de sección constante" coincide con él, $\mathscr{L}=\mathscr{A}$. Nótese que en un cable de tamaño $L=\mathscr{L}_{1}$ sin carga externa, la tensión en el extremo inferior es nula, siendo máxima en el superior $\sigma=\boldsymbol{f}$. La tensión media en el volumen es la mitad: no se aprovecha toda la resistencia del material.

Un cable $L<\mathscr{L}$ puede soportar carga útil [5]:

$$
Q=\boldsymbol{A f}\left(1-\frac{L}{\mathscr{L}}\right)=P\left(\frac{\mathscr{L}-L}{L}\right)
$$

'Para la historia de la ciencia, tiene interés notar como ni siquiera la mecánica se resistió a la ilusión de la reversibilidad -a pesar de la advertencia de GALIEO-, primero en la teoría -Euler da una solución al pandeo de barras bastante antes de que existieran soportes tan eficientes como para poder pandear-, después en la práctica, gracias al advenimiento de los nuevos materiales de "alta" resistencia y, por tanto, más "eficientes"... siempre que no pesaran ni se deformaran. 
con un coste y un rendimiento dados por [6]:

$$
C=\frac{\mathscr{A}}{\mathscr{A}-L}=\frac{\mathscr{L}}{\mathscr{L}-L} ; r=1-\frac{L}{\mathscr{A}}=1-\frac{L}{\mathscr{L}}
$$

Sobre esta sencilla expresión (4) puede construirse una teoría de diseño de estructuras semejantes, o análogas, si se entiende por diseño las reglas para obtener una estructura en el límite de economía (5). Desafortunadamente, esta sencilla medida del coste de un tamaño dado por su lejanía del insuperable no es exacta cuando la carga útil no es isomorfa con el peso propio, algo habitual en muchos otros esquemas estructurales; y tampoco se cuenta con una acotación consistente y general del error (2). Puesto que la expresión del coste bascula entre tamaños nulos y tamaños insuperables, por una parte se ha venido trabajando en la determinación de formas óptimas sin consideración del peso propio $(5,6,7)$; mientras por la otra se busca afinar la determinación del tamaño insuperable ligado a cada problema $(2,8,9,10)$; con ambos extremos razonablemente bien definidos puede aplicarse la regla [6] para estimar el rendimiento de una forma concreta para un tamaño dado, lo que permite la elección entre varias formas posibles para cada problema (para ejemplos concretos, véase 11).

\subsection{La catenaria}

El cable anterior suspendido entre dos puntos de una horizontal y sometido en exclusiva a su propio peso adopta como forma de equilibrio la curva denominada catenaria [7]:

$$
y=\frac{H}{\rho \boldsymbol{A}}\left\{\cosh \left(\frac{\rho \boldsymbol{A}}{H} x\right)-1\right\}
$$

siendo $H$ la reacción horizontal en los puntos de suspensión y, de hecho, la componente horizontal de la tracción en cualquier punto del cable. Puesto que el origen está en el punto más bajo de la curva, un cable que cubra una luz $\mathrm{L}$ tendrá un descuelgue, flecha o canto, $h=y(L / 2)$ [8]:

$$
h=\frac{H}{\rho \boldsymbol{A}}\left\{\cosh \left(\frac{\rho \boldsymbol{A}}{H} \frac{L}{2}\right)-1\right\}
$$

Es decir, para cada valor de $H$ se obtiene una curva de la familia infinita de curvas de equilibrio, con diferente valor de $h$. Es importante notar que las curvas de esta familia no son semejantes entre sí, ni geométrica ni mecánicamente, y que, por tanto, si buscamos la mejor de ellas nos adentramos fuera del campo de las soluciones semejantes, aunque seguimos manteniendo la sección constante del cable a lo largo de su longitud (como en el caso anterior).

El diseño de catenarias tiene que sortear el problema de que no es posible obtener $H$ en función de $h$ en forma analítica, siendo
$H$ imprescindible para obtener el esfuerzo máximo del cable y su área. La tracción en cualquier punto del cable es [9]:

$\mathbf{N}=H \cosh \left(\frac{\rho \boldsymbol{A}}{H} x\right)$

El esfuerzo máximo, en $L / 2$, vale [10]:

$\mathbf{N}=H \cosh \left(\frac{\rho \boldsymbol{A}}{H} \frac{L}{2}\right)$

Para un diseño en el límite de su resistencia se tendrá [11]:

$\mathbf{f} \boldsymbol{A}=H \cosh \left(\frac{\rho \boldsymbol{A}}{H} \frac{L}{2}\right)$

Denominando $\sigma_{\mathrm{o}}=H / \boldsymbol{A}$ a la tensión en el origen (el punto más bajo de la catenaria), la [11] puede escribirse en forma adimensional [12]:

$\frac{\boldsymbol{f}}{\sigma_{\mathrm{o}}}=\cosh \left(\frac{1}{2} \frac{\boldsymbol{f}}{\sigma_{\mathrm{o}}} \frac{L}{\mathscr{A}}\right) \Rightarrow \frac{L}{\mathscr{d}}=\frac{2}{p} \operatorname{acosh}(p)$

siendo $p=\boldsymbol{f} \div \sigma_{0}$. La condición para que $L$ sea máxima es [13]:

$\sqrt{p-1} \sqrt{p+1} \operatorname{acosh}(p)-p=0$

Su solución numérica es $p=1,8102(1 / p=$ $0,55243)$, resultando que [14]:

$L \leq \mathscr{L}=1,325$

En función de $p$, la definición geométrica de esa catenaria insuperable (de rendimiento nulo) es [15]:

$h(\mathscr{L})=\mathscr{A}\left(1-\frac{1}{p}\right)=0,45 \mathscr{A} \quad \lambda_{\mathscr{C}}=\frac{\mathscr{L}}{h(\mathscr{L})}=2,962$

Si el acero empleado es de alta resistencia con $\boldsymbol{f} \sim 500 \mathrm{~N} / \mathrm{mm}^{2}$, resulta que el alcance $\mathscr{A}$ es del orden de $6,37 \mathrm{~km}$ y la luz insuperable es mayor que 8 km. En 1985, el puente Akashi-Kaikyo en Japón con unos dos kilómetros de luz, alcanzó un 23\% del valor anterior [5], lo que podría considerarse un record. Pero en realidad tuvo que fabricarse un acero muy especial, con 1.200 $\mathrm{N} / \mathrm{mm}^{2}$ de tensión admisible para que su construcción fuera posible. Para ese acero, la luz del puente es tan sólo del $10 \%$ de su alcance. Tal parece que ese "record" del $10 \%$ del alcance estructural para el material usado en cada caso es una constante desde los primeros puentes de ferrocarril del siglo XIX. La razón es que para tamaños relativos mayores el coste (y otros problemas, fundamentalmente de rigidez) resultan insoportables. Es decir, hemos asistido a una mejora constante de la resistencia de los materiales (la primera vía de Galileo para sortear los tamaños insuperables) sin que, desafortunadamente, haya sido acompañada por una mejora igualmente generalizada de las formas estructurales. 
Puede parecer sorprendente que una catenaria pueda cubrir más luz que altura un cable suspendido. Pero no hay paradoja en ello: en un cable suspendido de tamaño insuperable la tensión media es la mitad de la máxima; en una catenaria insuperable la tensión varía desde $0,55 \boldsymbol{f}$ en el punto más bajo hasta $\boldsymbol{f}$ en el de suspensión, siendo su valor medio algo menor que $0,72 \boldsymbol{f}$ : una catenaria aprovecha mejor la resistencia del material que un cable vertical, aprovechándose para ello no solo de la reacción vertical de su sustentación, al igual que el cable, también de las reacciones horizontales, cuyo coste no estamos analizando aquí.

\section{SÓLIDOS DE TENSIÓN CONSTANTE}

Siguiendo la estela de Galileo, y una vez establecida la teoría de Bernouilu sobre la flexión de vigas, LAGRANGE intentó resolver el problema de obtener la mejor forma de una columna comprimida hacia 1770 . La primera solución en ese sentido fue dada dada por Clausen en 1851, y después por KeLLER (12), solución que ha sido posteriormente generalizada.

El problema de encontrar la forma de una ménsula cargada en el extremo con máxima capacidad de carga y mínimo coste, planteado por GALILEO, es un tema clásico en los manuales del siglo XX. Un recuento detallado de soluciones, incluyendo peso propio y diseño por resistencia o por rigidez, puede consultarse en Karihaloo et Hemp (13).

Para nuestros propósitos aquí, bastará con examinar con detalle la solución dada para el cable suspendido -simétrica de aquella otra para la columna, puesto que sus características más sobresalientes son las mismas que las formas propuestas para otros problemas (ménsula, etc).

\subsection{Un cable crecedero}

Si dejamos que la figura del cable suspendido adopte cualquier forma, dentro del esquema de una línea vertical, la variable adicional de diseño es su área, $A$. Puede plantearse la condición de que el cable tenga tensión constante bajo su propio peso [16]:

$\boldsymbol{f} A=\int_{0}^{L} \rho A d x ; \frac{d A}{A}=\frac{d x}{\mathscr{A}}$

y la solución es un perfil exponencial [17]:

$A=A_{0} \cdot \exp (x / \mathscr{A})$

y para una sección circular [18]:

$y=r_{0} \cdot \sqrt{\exp (x / \mathscr{A})}=r_{0} \cdot \exp \left(\frac{x}{2 \mathscr{A}}\right)$
Esta solución (Figura 1), parece superar los límites de la proposición VII: la tensión es constante con independencia del tamaño del cable. Y es capaz de soportar una carga útil $\left(Q=A_{0} \mathbf{f}\right)$.

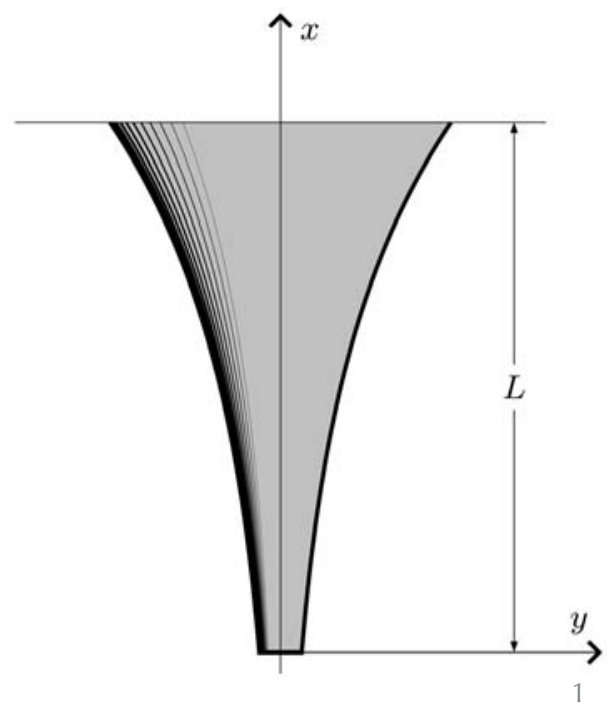

Pero el volumen del cable crece exponencialmente con su tamaño: $A_{0} / \mathscr{A}(\exp (L / \not)$ - 1), por lo que en cualquier caso es una estructura "intratable", con un coste [19]:

$C=\frac{Q+\rho V(L)}{Q}=1+\frac{1}{\rho}\{\exp (L / \mathscr{A})-1\}$

Además, desafortunadamente, no es la solución del problema planteado: la solución tan solo asegura la constancia de la tensión en la dirección de $x, \sigma_{x}=\boldsymbol{f}$. Pero ésta no es la tensión principal y como el borde está libre de tensiones, una dirección principal es normal a él $\left(\sigma_{b}=0\right)$ y la otra tangente. La tensión tangencial $\tau_{\mathrm{xy}}$ no es nula en el borde $y$, en consecuencia, la tensión normal en la dirección tangente al borde, $\sigma_{a}$, es mayor que $\boldsymbol{f}$ : la solución resulta inviable.

Más precisamente, $\sigma_{a}=\boldsymbol{f} / \cos ^{2}(\alpha)$ siendo $\alpha$ el ángulo de la tangente al borde con el eje $x$. Conforme $L$ crece, $\cos \alpha \rightarrow 0$ exponencialmente, y la tensión máxima crece de igual forma.

El crecimiento exponencial de la tensión o del coste no es de mucha importancia para la aplicación práctica de este tipo de soluciones: se aplican a tamaños estructuralmente pequeños $(L<<\mathscr{A})$ y, como es bien sabido, la función exponencial crece muy despacio cuando su argumento $(L / \mathscr{L})$ es pequeño: por tanto los errores en tales casos son despreciables y la aproximación muy buena.

Los problemas de esta solución no acaban ahí: para que con $\sigma_{x}=\boldsymbol{f}$ el tensor de tensiones sea admisible mecánica y cinemáticamente tiene que ocurrir (pensando en la versión 2D) que $\sigma_{y}=A+B y$ y $\tau_{x y}=-\rho x$ siendo $A$ y $B$ dos constantes de integración
1. Cable de tensión "constante": solución clásica. 
2. Simulación de una chapa "exponencial" a 400N/mm². De izquierda a derecha y de arriba abajo: $\sigma x, \sigma y, \tau x y$ y tensión de Von Mises. a determinar mediante condiciones de contorno, que, en este caso, pueden resumirse en que las direcciones principales de tensión sean paralelas al borde de la figura. De hecho, pueden determinarse tanto $A$ como $B$, pero $B$ tendría que ser una función no lineal de y, lo que impediría cumplir con la ecuación de compatibilidad en tensiones; al menos seguiría habiendo equilibrio, aunque $\sigma_{y}$ crecería exponencialmente con $y$. Por tanto, el cable crecedero no es un diseño de tensión constante.

No conocemos ninguna solución analítica para esta figura, y parece muy difícil obtenerla, de manera que no podemos decir nada acerca de la existencia de un tamaño límite. En la figura 2 puede observarse la fortísima concentración de tensiones en una chapa metálica 2D.

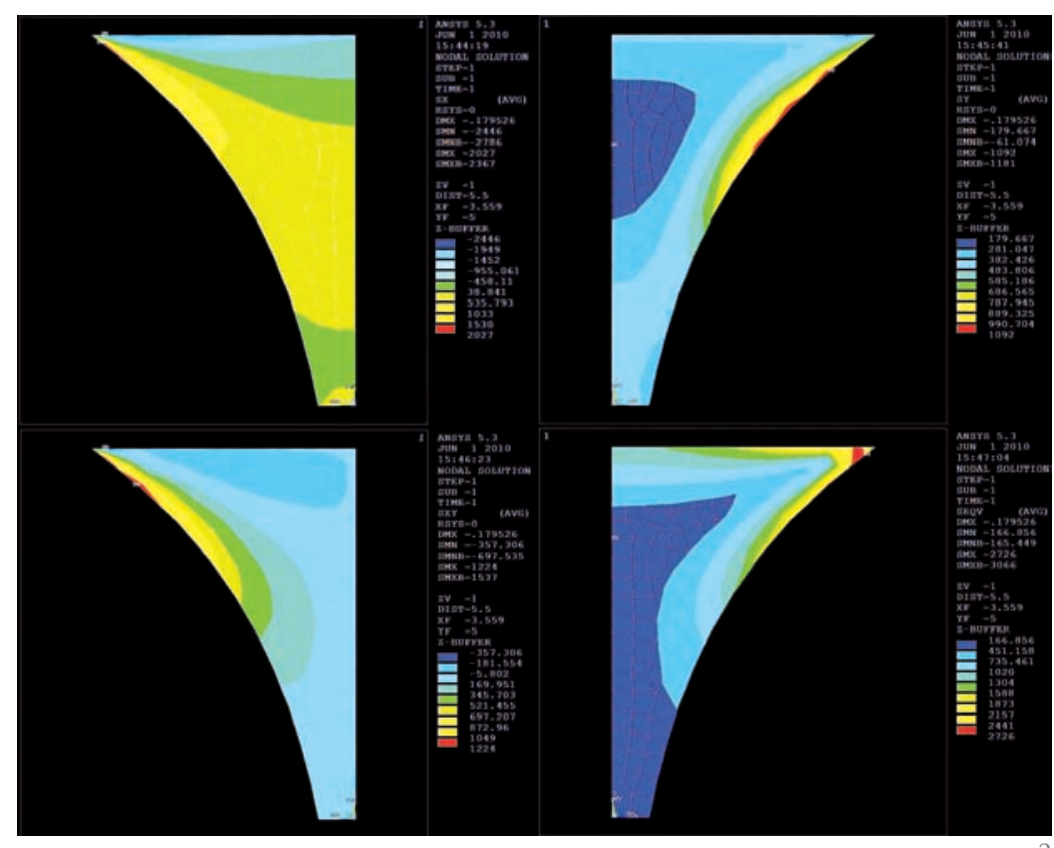

\subsection{Otras figuras crecederas}

Con los mismos problemas del cable crecedero, se conocen soluciones con tensión longitudinal constante para la ménsula y la viga doblemente apoyada sometidas a carga y a su propio peso (13). Como en el caso del cable no puede afirmarse nada respecto a la existencia de un tamaño insuperable: en todas ellas las tensiones principales crecen sin límite, aunque son útiles para tamaños pequeños $(14,15)$

Pero existen otras soluciones de tensión constante más cercanas a las estructuras reales: Cervera (10) presenta la de arco y arco + catenaria, de sección variable (exponencialmente) en ambos casos. Estas soluciones tienen problemas similares a las anteriores, tensiones principales de mayor valor que la tensión constante usada en el diseño. A pesar de ello, hay un tamaño límite insuperable que definen un alcance para ambos esquemas (aunque sea aproximadamente, por la inviabilidad de la solución). Más precisamente la luz máxima es $\pi /$ en ambos casos y, además, se trata de una longitud inalcanzable que requeriría una altura infinita.

\section{UNA HIPÓTESIS ADICIONAL}

Si se elige como hipótesis de trabajo a HP, el proceso de refutación es muy productivo: se trata de buscar aquellos casos en que es refutable $y$, por tanto, ir restringiendo su campo de validez. Por ello, durante la exploración, habrá que definir con más nitidez el contexto de los problemas estructurales en que HP pueda ser útil (estructuras sometidas a campos gravitatorios uniformes y otras acciones, criterios de rotura de los materiales, etc).

Dentro del campo de validez de HP, y para problemas concretos de interés, el otro objetivo prioritario es la determinación del tamaño insuperable para un problema concreto. De nuevo es necesaria una hipótesis de trabajo. A la vista de toda la exploración anterior cabe proponer la siguiente:

para cada problema, el tamaño insuperable para cualquier forma es el de la forma óptima de grueso constante

Por ejemplo, en el caso del problema de sostener un peso en el extremo inferior de un cable, el tamaño máximo para un cable de sección constante es el alcance estructural del material empleado. La hipótesis adoptada afirma que ningún otro cable de sección variable podrá superar ese límite. De nuevo se trata de una hipótesis refutable $y$, por tanto, podrá avanzarse en determinar su campo de validez. Aunque esta segunda hipótesis puede resultar sorprendente surge de la consideración de la tensión tangencial. Como es bien sabido, cualquier estado de tensión puede descomponerse en unas direcciones dadas en la suma de un estado de tensiones normales (sin tangenciales) y de otro de tensiones tangenciales (sin normales). El teorema de Michell y otras consideraciones sugieren que cualquier solución óptima tiene que estar libre de tensiones tangenciales en los puntos de tensión máxima. Finalmente, en cualquier elemento lineal, si la sección varía a lo largo de la directriz resulta inevitable la aparición de tensiones tangenciales a lo largo de todo el borde. Solo el grueso (sección) constante puede evitar su aparición. 


\section{CONCLUSIONES}

Hemos visto que existen casos en que la existencia de tamaños insuperables, y por tanto de problemas estructurales irresolubles, no puede ponerse en duda. Pero como solo se trata de algunos casos, no demuestran que HP sea generalizable a cualquier problema estructural. Además, como los casos presentados solo manejan estructuras de grueso constante, ni siquiera sirven para iniciar la "impracticable" refutación de $\mathrm{HO}$.

Por otro lado, existen pseudo soluciones muy prometedoras, y desde luego muy útiles para tamaños muy pequeños, que mal interpretadas pueden hacer creer que algunos problemas estructurales tendrían siempre solución con independencia del tamaño; pero tal creencia no sería más que una superstición. $\mathrm{HO}$, en consecuencia, no cuenta con ningún caso que la sostenga, aunque tampoco se han presentado refutaciones parciales concluyentes.

La cuestión, por tanto, permanece abierta. $Y$ en un contexto cultural como el presente, en el que la confianza en la bondad del "crecimiento indefinido" sigue siendo tan popular, nos parece que se trata de una cuestión de indudable interés cultural, a pesar de su apariencia teórica: si HP fuera cierta, podrían aportarse argumentos en contra de los modelos del "crecimiento" desde una disciplina como la mecánica, menos sospechosa de veleidades ideológicas que, por ejemplo, la ecología o el sentido común. De hecho, las estrategias a desarrollar para esa demostración podrían ser de utilidad en otros casos semejantes, la ecuación diferencial de transporte, por ejemplo.

De todas formas, demostrar HP no parece nada fácil; además podría tratarse de un teorema prescindible: a fin de cuentas, no parece que sea necesaria ninguna demostración para aceptar la certeza de que en un planeta finito no cabe un crecimiento indefinido de ninguna magnitud (aunque paradójicamente nuestra civilización acepta la hipótesis contraria con una naturalidad pasmosa).

La cuestión tiene otro interés añadido, más práctico: ver si es necesario mejorar el modelo estándar de la teoría de diseño, basada en relaciones simples entre tamaño y carga útil, como la que se dedujo en el caso del cable suspendido. Para ello sería necesario conocer el alcance de cada esquema estructural sin restringirnos a soluciones semejantes, buscando la forma óptima desde el punto de vista de mayorar el alcance estructural del esquema grueso constante.

\section{BIBLIOGRAFÍA}

(1) Cervera Bravo, J.: Tres teoremas fundamentales de la teoría del diseño de estructuras. Informes de la Construcción, vol. 40, num. 399, pp. 57-66, 1989.

(2) Cervera Bravo, J.: Las estructuras y el peso propio. Informes de la construcción, vol. 42, núm. 407, pp. 73-86, 1990.

(3) Kauffman, S.: Investigations. Oxford University Press, New York, 2000

(4) Rankine, W. J. M.: A manual of civil engineering. $2^{\text {a }}$ edición, 1863.

(5) Michell, A. G. M.: The Limits of Economy of Material in Frame-structures. Philosophical Magazine, S.6, vol. 8, num. 47, pp. 589-597, 1904.

(6) Vázquez Espí, M.: Un nuevo algoritmo para la optimación de estructuras: el recocido simulado. Informes de la Construcción, vol. 46, núm. 436, pp. 46-ᄀ69, 1995.

(7) Cervera Bravo, J.: Concebir y analizar estructuras. Universidad Politécnica de Madrid, V2.0, 218 pp.

(8) Cox, H. L.: The design of structures of least weight. Pergamon, Oxford, 1965.

(9) Hemp, W. S.: The theory of structural design. Cranfield: College of Aeronautics, Report 115. 1958.

(10)Cervera Bravo, J.: Diseño de estructuras en edificación. Instituto Juan de Herrera, Madrid, 1993. http://oa.upm.es/3785/

(11) Fernández Cabo, J. L.: Estructura: tamaño, forma y proporción. Tesis doctoral. Madrid: Universidad Politécnica de Madrid. 1998.

(12) Keller, J. B.: The shape of the strongest column. Arch. ration. Mech. Analysis. Vol. 5, pp. $275-285$. 1960.

(13) Karihaloo, B. L.; Hemp, W. S.: Maximum stregth/stiffness design of structural members in presence of self-weight. Proc. R. Soc. Lond. A, vol. 389, pp. 119-32. 1983.

(14) Chai, Y. H.; Wang, C. M.: Approximate Solution for the Shape of Submerged Funicular Arches with Self-weight Journal of Structural Engineering, vol. 131, num. 3, pp. 399-404. 2005.

(15) Zheng, B.; Ching-jui Chang, C.; Hae Chang Gea, H. C.: Topology Optimization Considering Body Forces. International Journal for Simulation and Multidisciplinary Design Optimization. Vol. 3, pp. 316-320. 2009. 